\title{
Growing Large Size Complex Oxide Single Crystals by Czochralski Technique for Electronic Devices
}

\author{
I.M. SOLSKII ${ }^{a, *}$, D.YU. SUGAK ${ }^{a, b}$ AND M.M. VAKIV ${ }^{a, b}$ \\ ${ }^{a}$ Scientific Research Company "Carat", 202, Stryjska st., Lviv, 79031, Ukraine \\ ${ }^{b}$ Lviv Polytechnic National University, 12, Bandera st., Lviv, 79013, Ukraine
}

\begin{abstract}
This paper is devoted to the use and further development of the single crystal growing technique invented by Professor Jan Czochralski (1885-1953). The possibilities of the Czochralski technique are demonstrated. Further improvements were introduced at the Scientific Research Company "Carat" (Lviv, Ukraine) to grow large size complex oxide single crystals. The paper presents an overview of some single crystals grown with the use of a modified technology. Growth parameters and properties of resulting crystals are summarized to show a high potential of the Czochralski technique as an industrial technology to grow large-size, high quality, and structurally perfect single crystals of complex oxides.
\end{abstract}

DOI: 10.12693/APhysPolA.124.314

PACS: 81.10.-h

\section{Introduction}

Since the 1970s the electronic industry has been facing a strong demand for novel devices based on new physical principles. Examples are electro- and acousto-optic modulators and $Q$-switches, acoustoelectric delay lines, surface-acoustic-wave filters (SAW filters); devices of nonlinear, integral and polarization optics, new laser active media, as well as scintillation detectors for environmental monitoring, tomography, and high-energy physics [1-8]. Such devices are mainly based on the use of complex oxide single crystals with unique physical properties. Thus, the development of a reliable technology to grow large size single crystals of oxide compounds has become a vital task.

During the last few decades, new microelectronic components and new-generation processing facilities are able, precisely to operate at melting temperatures up to $2800 \mathrm{~K}$ have become available. This makes the stability of growth thermal environment and other processing parameters a true challenge which can be answered through further improving the Czochralski technique.

The Czochralski method has been widely used to grow complex oxide single crystals since the 1960s. Over this period, the technique keeps being improved, and a variety of crystals grown by the Czochralski method is extending. The method history is described in a number of review articles.

The review [9] was one of the first publications to discuss the general issues and a scope of application of the method and to consider which types of crystals can be grown. The Czochralski technique is most used to fabricate laser crystals and laser beam control crystals. The experience of Polish scientists in this field is summarized

*corresponding author; e-mail: solskii@carat.lviv.ua in [10]. For example, the paper not only describes routine technologies to grow "traditional" activated garnet and vanadate crystals, but also considers how the Czochralski method can be used to grow complex borate crystals doped with rare-earth elements which have both laser and nonlinear optical properties.

In 2003, in Ref. [11] it was reported that the method was used to grow new laser crystals with the structure of olivine and phenacite doped with $d$-elements.

The review [12] is of special interest since it considers the history of the Czochralski method development and its status as of 2004, compares its benefits and disadvantages, and indicates the directions of further improvement. Thus, a special focus is placed on increasing the size of resulting crystals, extending a variety of crystal types, implementing high frequency heating of a crucible, improving the diameter control of growing crystal. The weighing method was considered the most promising one among other diameter control methods.

\section{Specifics of using the Czochralski technique}

The principle of this technique implies melting a starting raw material in a platinum or iridium crucible (this condition is mandatory because the oxide compound melts are aggressive to any substance except refractory noble metals) and dipping a seed, previously oriented with X-ray method, into the melt followed by pulling out a single crystal at a given rate, with the crystal being rotated.

Since the melting temperatures of most oxide compounds exceed $1800 \mathrm{~K}$, the Czochralski process to grow single crystals is performed with the use of high frequency heating equipment of GALAXIE type which was specially designed for the SRC "Carat" by PHYSITERM (France) and then modified by "Carat" specialists.

The single crystal is grown in a sealed chamber with a water-cooled case. Thermal environment around the crucible creates the thermal gradients necessary for growing 
and annealing the single crystal after the growth is completed.

The crucible is heated up by $24 \mathrm{kHz}$ high frequency generator radiation. The computer-assisted weighing method is used to control the crystal diameter during the growth. A tensoresistive bridge placed on a rod with a seed is a measuring element. The software ensures the process of $\mathrm{PC}$ visualization at all stages to begin from the time of nucleation and up to separation of the crystal. Figure 1 shows a control PC screenshot presenting the real process parameters and the growth stages of a lithium niobate crystal.

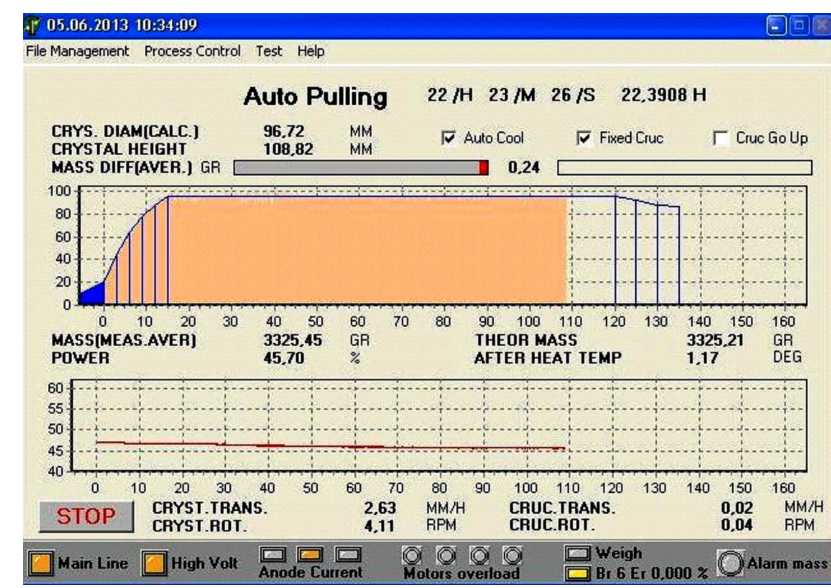

Fig. 1. Process parameters and the growth stages of a lithium niobate crystal on a control PC screenshot.

The pulling rate, depending on the crystal type and the growing stage, can vary within $0-100 \mathrm{~mm} / \mathrm{h}$, and the rotation rate - within $0-100 \mathrm{rpm}$. The equipment is shown in Fig. 2.

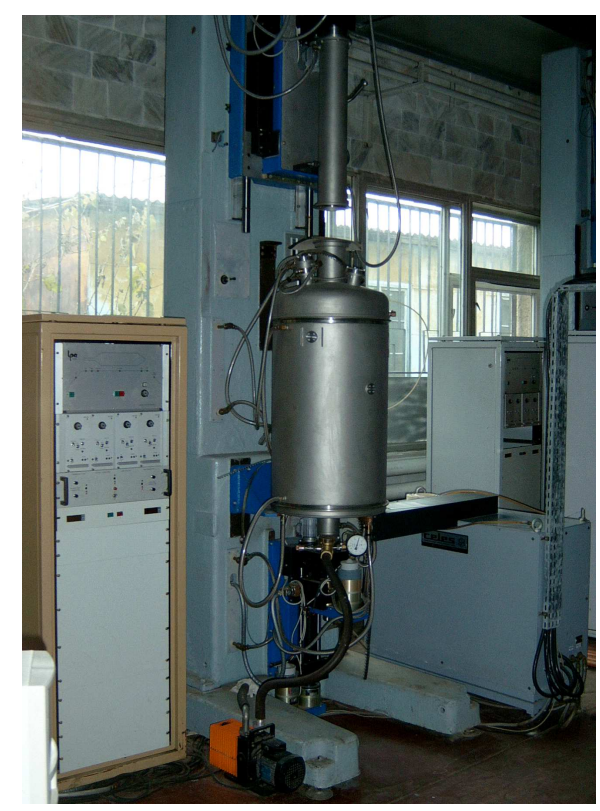

Fig. 2. Crystal growth equipment.
A special feature of the Czochralski growth facilities at SRC "Carat" is that they were originally designed to produce large size single crystals up to $40 \mathrm{~kg}$ and up to $110 \mathrm{~mm}$ in diameter. This imposes special requirements to the thermal environment since large size crystals require large size crucibles, with the ratio of a resulting crystal diameter to a crucible diameter (and the diameter of melt surface) being about 1:2. We should also note that the diameter of an inductive coil in growth units at the SRC "Carat" allows installing a crucible of $250 \mathrm{~mm}$ external diameter inside the coil. This makes it possible to grow complex oxide crystals up to $125 \mathrm{~mm}$ in diameter.

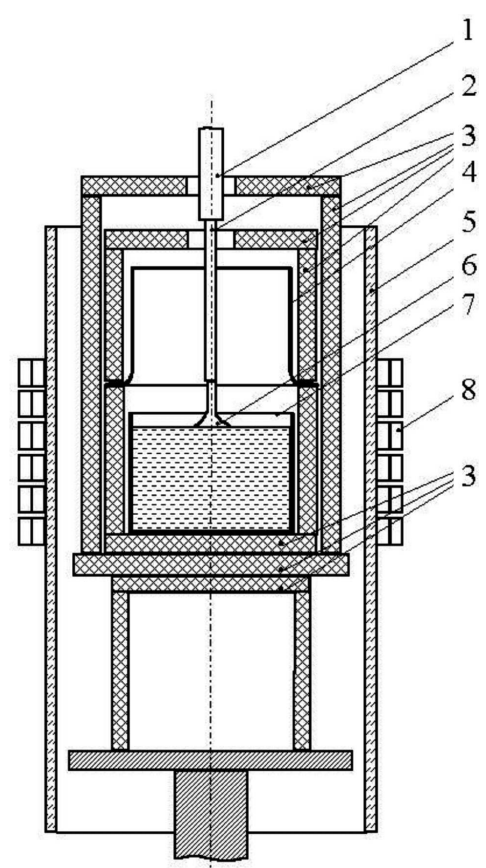

Fig. 3. A typical thermal unit: 1-3 - parts made of special corundum and yttrium-zirconium ceramics, $4-$ iridium or platinum screen, 5 - quartz tube, 6 - oriented seed, 7 - iridium or platinum crucible, 8 - inductor coil.

Thus, for each type of single crystals, in order to ensure their optical quality and structural perfection, the growth interface should be made as flat as possible through controlling radial thermal gradients as well as crystal pulling and rotational rates. In addition, in each specific case a small overcooling is necessary for maintaining the specified vertical thermal gradients to ensure the reliable crystal growth and gradual cooling of a crystal part above a melt. For the large size crystals, designing a thermal unit of a growth chamber becomes a special problem. Figure 3 presents a typical thermal unit, with elements 1-3 being made of special corundum and yttrium-zirconium ceramics to ensure refractory and thermal insulation and maintain the thermal radial gradient, 4 - an iridium or platinum screen, its dimension and position relative to the crucible edge allow influencing the thermal environment above the melt, 5 - a quartz tube which is both 
an additional thermal insulator and an electric one, 6 a properly oriented seed, 7 - an iridium or platinum crucible, 8 - an inductor coil to induce whirling currents which heat the crucible up to a required temperature. This typical design is customized to each single crystal.

Another important factor influencing the quality of single crystals is the purity of starting raw materials. Therefore they are prepared in the form of fine powders with a controlled amount of impurities and 99.99-99.999\% of the main phase. At the first stage (charge preparation) these powders are mixed in stoichiometric proportions, subjected to high temperature synthesis in a solid phase and tableted.

Then the tablets are placed into the crucible where the raw material is deposited, and the melt is further homogenized at the temperature that exceeds the raw material melting temperature. In fact, the growth starts at this moment.

\section{Application and properties of resulting single crystals}

Several manufacturing processes were developed to grow a large range of complex oxide single crystals using the technology described above.

Growth parameters are summarized in the following Table.

Growth parameters.

TABLE

\begin{tabular}{c|c|c|c|c|c}
\hline \hline Crystal & $\begin{array}{c}\text { Melting } \\
\text { temperature } \\
{[\mathrm{K}]}\end{array}$ & $\begin{array}{c}\text { Crystallographic } \\
\text { growth direction } \\
{[h k l]}\end{array}$ & $\begin{array}{c}\text { Content } \\
\text { of activator } \\
\text { in a crystal } \\
\text { [at.\%] }\end{array}$ & $\begin{array}{c}\text { Crystal growth } \\
\text { and rotational rates } \\
{\left[\mathrm{mm} \mathrm{h}^{-1} / \text { rev min }^{-1}\right]}\end{array}$ & $\begin{array}{c}\text { Crystal } \\
\text { dimensions } \\
{[\mathrm{mm}]}\end{array}$ \\
\hline $\mathrm{Gd}_{3} \mathrm{Ga}_{5} \mathrm{O}_{12}$ & 2013 & {$[111]$} & - & $7 / 15-22$ & $\varnothing=82-85, L \leq 350$ \\
$\mathrm{Gd}_{3} \mathrm{Ga}_{5} \mathrm{O}_{12}: \mathrm{Nd}$ & 2013 & {$[111]$} & 1.7 & $7 / 15-22$ & $\varnothing=60, L \leq 100$ \\
$\mathrm{LiNbO}_{3}$ & 1523 & {$[0001]$} & - & $5 / 6-8$ & $\varnothing=96, L=110$ \\
$\mathrm{YAlO}_{3}: \mathrm{Nd}$ & 2140 & {$[010], P_{b n m}$ set } & 1.4 & $3 / 15-18$ & $\varnothing=65, L \leq 140$ \\
$\mathrm{YAlO}_{3}: T m$ & 2140 & {$[010], P_{b n m}$ set } & 4.2 & $3 / 15-18$ & $\varnothing=60, L \leq 110$ \\
$\mathrm{CaWO}_{4}$ & 1849 & {$[110]$} & - & $3 / 15-18$ & $\varnothing=60-65, L=140$ \\
$\mathrm{CdWO}_{4}$ & 1643 & {$[100]$ or $[010]$} & - & $3 / 15-18$ & $\varnothing=60-65, L \leq 170$ \\
$\mathrm{CaMoO}_{4}$ & 1703 & {$[100]$ or $[010]$} & - & $3 / 18-22$ & $\varnothing=55-60, L=140$
\end{tabular}

The values of growth and rotational rates presented in Table were determined in the course of numerous technological experiments followed by testing the optical and structural perfection of resulting crystals. When a thermal unit has been made and cannot be re-designed, it is only growth and rotational rates that provide practically a single way to influence a shape of the growth interface during the crystal growth.

Thus, Brandle [12] rightly pointed out that the problem of designing a thermal unit and choosing parameters of growth does not have a single solution, but requires experimenting. It should be noted that indicated parameters depends on physical-chemical properties of the material in the solid and liquid phases as well as on the sizes of resulting crystals.

Properties of resulting crystals and their application are discussed in the next sections.

\subsection{Gadolinium gallium garnet}

$\mathrm{Gd}_{3} \mathrm{Ga}_{5} \mathrm{O}_{12}$ (GGG) single crystals are employed as substrates for liquid-phase epitaxial deposition of single crystal magnetic ferrum-yttrium garnet thin films and $\mathrm{Gd}_{3} \mathrm{Ga}_{5} \mathrm{O}_{12}: \mathrm{Cr}^{4+}$ single crystal thin films to be used as passive $Q$-switches of solid-state neodymium lasers $[13,14]$. Structural perfection of GGG single crystals should be very good because substrate structural defects are reproduced in monocrystalline thin films during the epitaxial growth. Our technologies allow growing practically defect-free GGG crystals which do not have mechanical stresses, with the density of dislocations in substrates being below $0.5 \mathrm{~cm}^{-2}[15]$. Epitaxial growth technologies to produce a wide range of magnetic thin films and laser film media of garnet structure were also developed. A GGG crystal is shown in Fig. 4.

Another important application of GGG:Nd single crystals are active elements of 1.062 and $1.44 \mu \mathrm{m}$ solid-state lasers. Though GGG:Nd single crystals have slightly worse thermophysical characteristics than the most widely used $\mathrm{Y}_{3} \mathrm{Al}_{5} \mathrm{O}_{12}$ :Nd (YAG), they have a number of advantages such as better manufacturability and a greater activator distribution coefficient 0.4 [16]. GGG:Nd-based lasers successfully operate in pulse-periodic modes with a low pulse repetition rate. The characteristics of lamp- and diode-pumped lasers with active elements made of resulting crystals are given in $[17,18]$.

High quality of resulting GGG:Nd crystals also has allowed manufacturing active elements in the form of slabs with the dimensions of $6 \times 8 \times 115 \mathrm{~mm}^{3}$ and the Brewster-angled working surfaces. Tests carried out at "EDAPS- 


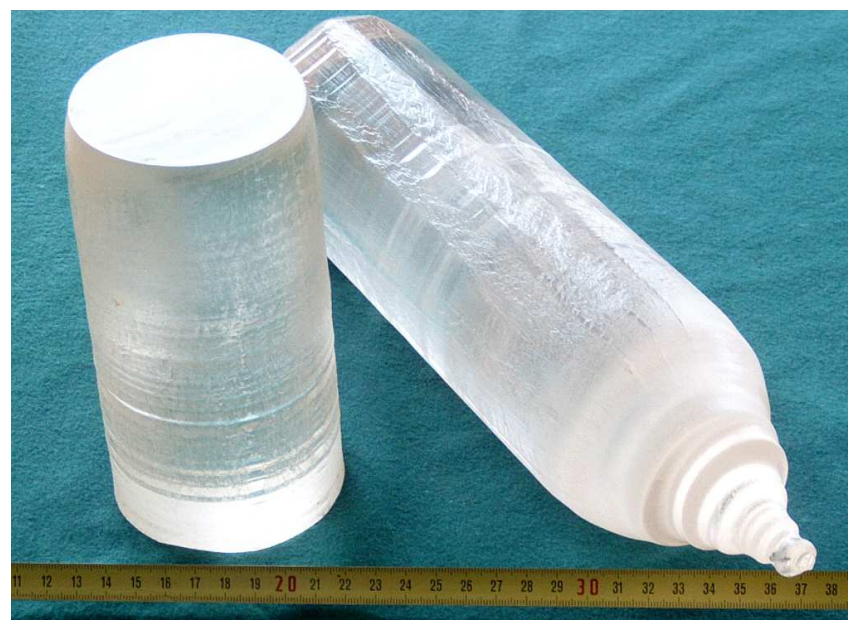

Fig. 4. Gadolinium gallium garnet single crystal.

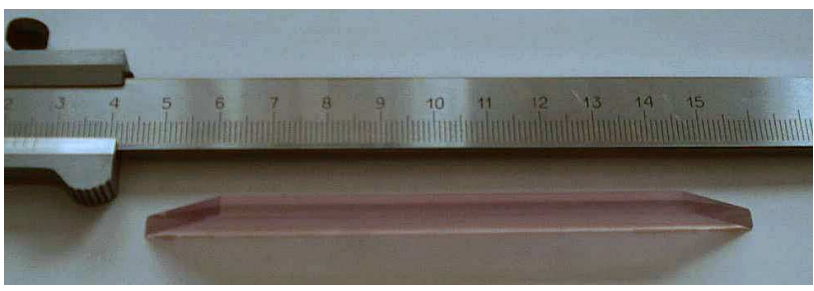

Fig. 5. The laser slab made of the GGG:Nd single crystal.

-Laser Ltd" (Kyiv, Ukraine) have demonstrated that, when using a modified diode pumping ZIGZAG scheme (a total pumping radiation power being approximately $200 \mathrm{~W}$ ) and passive $Q$-switching, the laser can reach a power of several tens $\mathrm{kW}$ in the $10 \mathrm{~ns}$ pulse [19]. Figure 5 presents a slab made of the GGG:Nd single crystal.

\subsection{Yttrium aluminum perovskite}

The primary advantage of the YAP crystal, compared to YAG, is better manufacturability - its melting temperature $(2140 \mathrm{~K})$ is lowered by eighty degrees, and the growth rate is $3-5$ times higher. Also, the impurity distribution coefficient is higher, compared to YAG. Thus, for $\mathrm{Nd}$ it equals 0.8 and 0.18 , respectively [20]. For Tm, similar values are unknown; however we can expect that they are nearly the same for all lanthanides.

Additionally to better manufacturability, YAP:Nd crystals have another advantage over YAG:Nd - it is higher luminescence efficiency at the wavelength of $1.34 \mu \mathrm{m}$ [21] that makes no harm to human retinal tissues. Such lasers have found wide application in the environmental monitoring devices and range-finders.

Nowadays the YAP:Tm crystal is considered one of the best laser materials that generate in the region of $2 \mu \mathrm{m}$ of wavelengths [22]. Laser radiation in this region is widely used in military equipment [23], meteorology, and location [24]. The range of its medical applications is
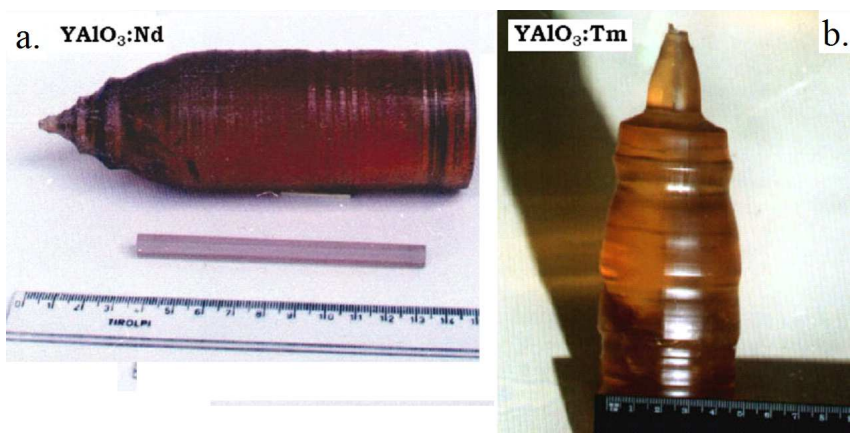

Fig. 6. Yttrium aluminum perovskite single crystals: (a) YAP:Nd, (b) YAP:Tm.

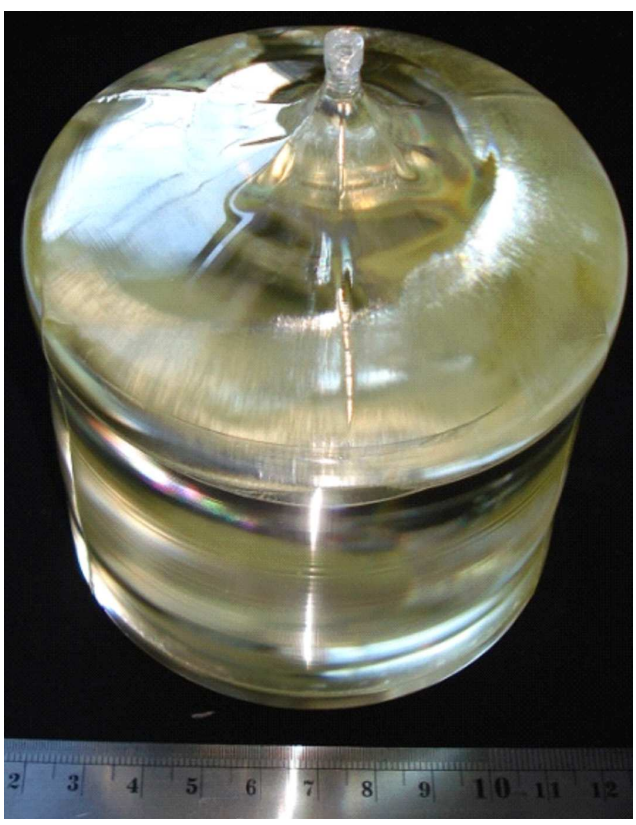

Fig. 7. Lithium niobate single crystal.

particularly wide - surgery, ophthalmology, gynecology, orthopedics, arthroscopy, angioplasty, and others [23, 24]. The special features enabling the use of radiation at this wavelength are strong absorption by water and human body tissues, correspondingly; weak absorption in the atmosphere, and safety for the human eye.

The processes described in $[25,26]$ were applied to grow YAP:Nd and YAP:Tm to make the rod-like active elements (YAP:Nd) for lamp-pumped lasers and wafer-like active elements (YAP:Tm) for diode pumping. The efficiency of the laser with a YAP:Nd active element at the wavelength of $1.079 \mu \mathrm{m}$ was equal to $3 \%$, the differential efficiency of the $1.936 \mu \mathrm{m}$ YAP:Tm laser with diode pumping at the wavelength of $792 \mu \mathrm{m}$ was equal to $54 \%$ $[27,28]$.

Figure 6 presents YAP:Nd and YAP:Tm crystals.

\subsection{Lithium niobate}

Using the active elements of electro-optic and acousto-optic laser radiation control devices made of $\mathrm{LiNbO}_{3}$ sin- 
gle crystals requires a high optical homogeneity of crystals. It is desirable to have large crystals to be able to make as many ready-to-use optical elements as possible from one ingot. To address these needs, a technology has been developed to perform growing, single-domaining and quality control of large-size lithium niobate single crystals of high optical homogeneity [29]. Our approach has allowed growing the single crystal boules of $95 \mathrm{~mm}$ in diameter, with a cylindrical part being longer than $100 \mathrm{~mm}$. The two thirds of the resulting boule have the value of anomalous birefringence $\Delta n_{\mathrm{a}}$ not exceeding $10^{-5}$ per $1 \mathrm{~cm}$ of crystal length. The resulting crystals are used to fabricate the active elements of electro-optic and acousto-optic $Q$-switches for solid-state laser cavities, and the substrates for various acoustoelectronic devices, including radio signal SAW filters. Figure 7 shows a lithium niobate single crystal.

\subsection{Cadmium and calcium tungstates and calcium molybdate}

Practical requirements of high energy experimental physics and computer-assisted tomography have stimulated interest to developing new scintillation detectors based on oxide crystals for their high efficiency in X-ray and $\gamma$-ray registration and high radiation and chemical resistance.

Cadmium tungstate $\mathrm{CdWO}_{4}$ (CWO) is a promising medium for creating the scintillation detectors of total absorption of $\gamma$-quanta ( $\gamma$-radiometers for environmental monitoring, $\gamma$-ray logging in geology), and detectors for X-ray CT-scanners $[7,30]$.

A specific feature of growing $\mathrm{CdWO}_{4}$ crystals is a partial loss of cadmium oxide that deteriorates optical and scintillation properties of the crystals. To avoid such an effect, $\mathrm{CdCO}_{3}$ is added to the stoichiometric raw material in an amount ensuring that the excess of $\mathrm{CdO}$ is equal to $1.0-1.1 \mathrm{wt} \%$. The resulting CWO crystals are practically colorless within the cylindrical part (up to $140 \mathrm{~mm}$ ), and have the quantum yield of $36.5 \%$ with regard to NaI:Tl and the energy resolution of $\approx 9 \%$ in case of exciting by a ${ }^{137} \mathrm{Cs} \gamma$-quantum source $(662 \mathrm{keV})$. A detailed description of the technology for growing large-size CWO crystals is given in [31]. A CWO crystal is shown in Fig. 8.

Calcium tungstate $\mathrm{CaWO}_{4}$ (CaWO) is another representative of the tungstate class; its scintillation characteristics can potentially exceed the CWO level in terms of quantum yield and energy resolution, while fully conserving the efficiency of registering the ionizing radiation [32]. Particular attention should be drawn to a lower cost and better environmental safety of manufacturing CaWO (compared to CWO). Large size $\mathrm{CaWO}_{4}$ single crystals have been grown from the iridium crucibles using argon. Anisotropy of thermal expansion coefficients of CaWOcan result in formation of cracks in the cleavage plane (001) during the growth or post-growth annealing. Therefore, in order to preserve the crystal integrity, the growth was carried out perpendicularly to the $z$-axis in the direction [110]. The resulting CaWO single crystals

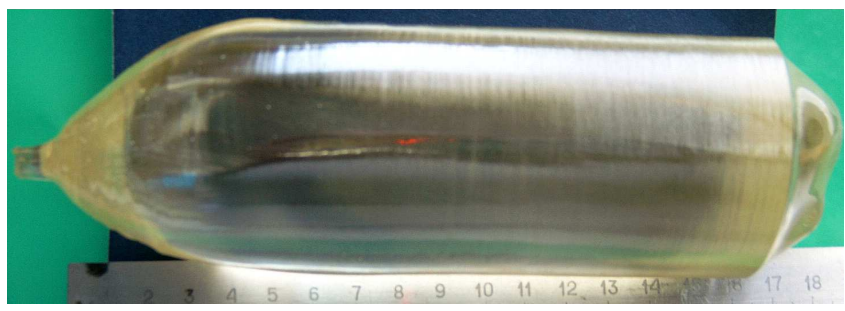

Fig. 8. Cadmium tungstate single crystal.

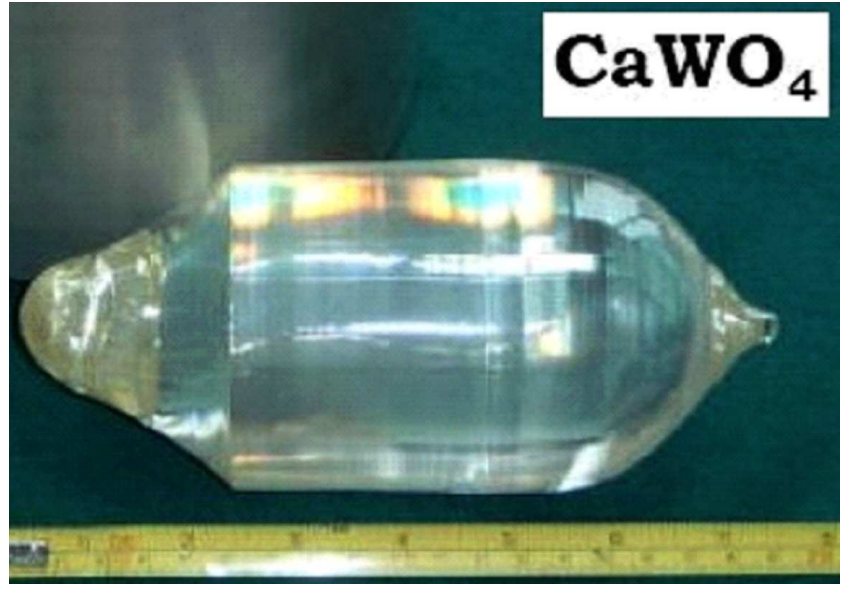

Fig. 9. Calcium tungstate single crystal.

are typically grey, but this coloring totally disappears after annealing in oxidizing ambient at a temperature of $\approx 1520 \mathrm{~K}$. The light yield of $\mathrm{CaWO}_{4}$ scintillators in case of exciting by ${ }^{137} \mathrm{Cs} \gamma$-quantum source $(662 \mathrm{keV})$ is by $30-40 \%$ higher than in $\mathrm{CdWO}_{4}$ samples of similar dimensions.

The growth technology is described in [33], and the resulting crystals are shown in Fig. 9.

The arguments to develop $\mathrm{CaMoO}_{4}$ are as follows: ${ }^{100} \mathrm{Mo}$ is one of the most promising candidate to search for neutrinoless double beta decay (a key process in the modern physics) [34]; $\mathrm{CaMoO}_{4}$ contains valuable part of $\mathrm{Mo}(48 \%)$; $\mathrm{CaMoO}_{4}$ does not contain radioactive elements; $\mathrm{CaMoO}_{4}$ is a reasonably efficient scintillator even at room temperature [35]; $\mathrm{CaMoO}_{4}$ is a promising material for low temperature scintillating bolometers (with high energy resolution and particle discrimination ability) $[36,37]$.

The calcium molybdate single crystals were grown from previously synthesized raw materials in platinum crucibles in air. It should be taken into account that $\mathrm{MoO}_{3}$ evaporation is possible during the synthesis that causes the formation of defects. The best possible values of optical transparency and light output are ensured by growing the crystals from recrystallized raw materials with 1.0 wt\% excess of $\mathrm{MoO}_{3}$ in a charge. The growth direction [001] lies in the intense chipping plane.

An important feature of the crystal is that its grow- 

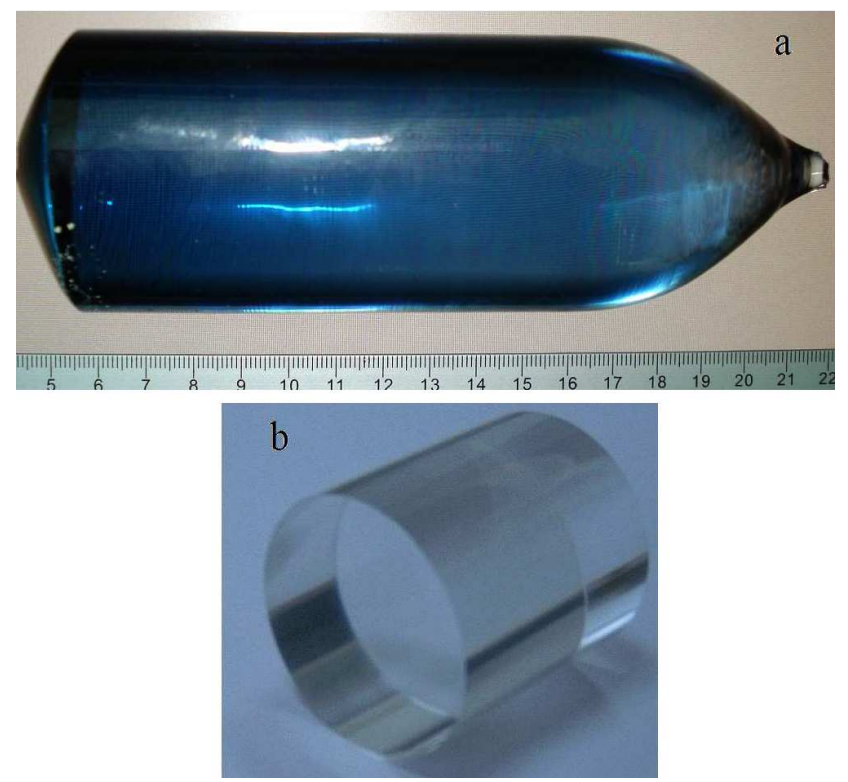

Fig. 10. Calcium molybdate single crystal: (a) a resulting blue crystal, (b) a colorless crystal after annealing in $\mathrm{O}_{2}$.

ing is accompanied by $\mathrm{Mo}^{6+}$ reduction, and the crystals become blue. Adding an extra amount (with respect to stoichiometry) of molybdenum trioxide to the raw material can slightly decrease the color intensity. Crystal annealing in oxygen will completely remove the coloration. The growth technology is detailed in [38]. Resulting and annealed crystals are given in Fig. 10.

\section{Conclusions}

Thus, our experience and research findings provide the evidence that the Czochralski technique in its current form is one of the most common industrial methods that allows to grow large-size, high quality, and structurally perfect single crystals of complex oxides to be used in functional electronic devices.

While comparing SRC "Carat" technological potential to grow complex oxide single crystals by the Czochralski method with achievements of internationally renowned research and industrial companies and institutions, we would like to note that SRC "Carat" is a leader today among similar enterprises in the Former Soviet Union for diversity and sizes of resulting crystals. Sizes and quality of our crystals can be compared with those of crystals produced by such industrial leaders as: Gooch \&Housego Palo Alto, USA (former Crystal Technology, Inc.) [39], Yamaju Ceramics Co., Ltd., Japan [40], Zhejiang Jinsheng Optoelectronics Co., Ltd, China [41] — lithium niobate, Institute for Crystal Growth (IKZ), Germany [42] — rare-earth gallium garnets, Crytur, Czech Republic [43] and Institute of Electronic Materials Technology, Poland [44] — yttrium aluminum perovskites.

\section{References}

[1] Acoustic Crystals. Handbook, Ed. M.P. Shaskolskaya, Nauka, Moscow 1982 (in Russian).

[2] A.I. Vaniurikhin, V.P. Gerchanovskaya, Optoelectronic Polarization Devices, Tekhnika, Kiev 1984 (in Russian).

[3] V.I. Balakshij, V.N. Parygin, L.E. Chirkov, Physical Basis of Acousto-optics, Radio i sviaz', Moscow 1985 (in Russian).

[4] A.A. Kaminskii, Crystalline Lasers: Physical Processes and Operating Scheme, CRC Press, Boca Raton 1996.

[5] Yu.S. Kuz'minov, Lithium Niobate Crystals, Cambridge International Science Publishing, Cambridge 1997.

[6] D.N. Nikogosyan, Properties of Optical and Laser Related Materials, Wiley, Chichester 1997.

[7] M. Globus, B. Grinyov, J.K. Kim, Inorganic Scintillators for Modern and Traditional Applications, Institute for Single Crystals, Kharkov 2005.

[8] T. Volk, M. Wöhlecke, Lithium Niobate: Defects, Photorefraction and Ferroelectric Switching, Springer, Berlin 2008.

[9] K. Nassau, A.M. Broyer, J. Am. Ceram. Soc. 45, 474 (1962).

[10] T. Lukasiewicz, A. Majchrowski, Z. Mierczyk, OptoElectron. Rev. 9, 49 (2001).

[11] T.F. Veremeichik, E.V. Zharikov, K.A. Subbotin, Cryst. Rep. 48, 974 (2003).

[12] C.D. Brandle, J. Cryst. Growth 264, 593 (2004).

[13] Components and Devices for Magnetic Bubble. Handbook, Eds. N.N. Yevtikhiev, B.N. Naumov, Radio i sviaz', Moscow 1987 (in Russian).

[14] A.O. Matkovskii, I.M. Syvorotka, S.B. Ubizskii, S.S. Melnyk, M.M. Vakiv, I.I. Izhnin, Tekhnologiya $i$ konstruirovanie v elektronnoi apparature 3, 1 (2002) (in Russian).

[15] Yu.N. Gavriliuk, A.G. Kuzmik, A.O. Matkovskii, B.K. Ostafijchuk, V.M. Pylypiv, I.M. Solskii, V.D. Fedoriv, P.I. Shevchuk, V.N. Shevchenko, Defects in Gadolinium Gallium Single Crystals, Institute of Metal Physics, Kiev 1989.

[16] D.L. Sun, Q.G. Zhang, Z.B. Wang, J. Su, C.J. Gu, A.H. Wang, S.T. Yin, Cryst. Res. Technol. 40, 698 (2005).

[17] D. Sugak, A. Matkovskii, A. Durygin, A. Suchocki, I. Solskii, S. Ubizskii, K. Kopczynski, Z. Mierczyk, P. Potera, J. Lumin. 82, 9 (1999).

[18] D.Yu. Sugak, B.M. Kopko, V.G. Haiduchok, I.I. Syvorotka, I.M. Solskii, A.P. Luchechko, M.M. Vakiv, O.A. Buryy, Solid State Phenom. 200, 181 (2013).

[19] G.B. Sidorov, "EDAPS-Laser" Ltd., private communication, Kiev 2011.

[20] A.O. Matkovskii, Materials for Quantum Electronics, Liga-Press, Lviv 2000 (in Ukrainian).

[21] Y. Wei, G. Zhang, C.-H. Huang, H.-Y. Zhu, L.-X. Huang, G.-F. Wang, Opt. Commun. 283 , 5153 (2010).

[22] I.F. Elder, J. Payne, Appl. Opt. 36, 8606 (1997). 
[23] C. Li, J. Song, D. Shen, N. Seong Kim, K.-I. Ueda, Y.J. Huo, S.F. He, Y.H. Cao, Opt. Expr. 4, 12 (1999).

[24] I.F. Elder, M.J.P. Payne, Opt. Commun. 148, 265 (1998).

[25] D.I. Savytskii, L.O. Vasylechko, A.O. Matkovskii, I.M. Solskii, A. Suchocki, D.Yu. Sugak, F. Wallrafen, J. Cryst. Growth 209, 875 (2000).

[26] A.O. Matkovskii, D.I. Savytskii, D.Yu. Sugak, I.M. Solskii, L.O. Vasylechko, Ya.A. Zhydachevskii, M. Mond, K. Petermann, F. Walraffen, J. Cryst. Growth 241, 455 (2002).

[27] D.Yu. Sugak, A.O. Matkovskii, V.V Grabovskii, V.I. Prokhorenko, A. Suchocki, A.M. Durygin, I.M. Solskii, A.P. Shakhov, Acta Phys. Pol. A 93, 643 (1998).

[28] D. Sugak, A. Dyrugin, A. Matkovskii, A. Suchocki, I. Solskii, D. Savytskii, Ya. Zhydachevskii, F. Walraffen, K. Kopczynski, Cryst. Res. Technol. 36, 1123 (2001).

[29] I.M. Solskii, D.Yu. Sugak, V.M. Gaba, Tekhnologiya konstruirovanie $v$ elektronnoi apparature 5, 51 (2005) (in Russian).

[30] Yu.A. Tsirlin, M.E. Globus, E.P. Sysoyeva, Optimization of $\gamma$-Radiation Detection with Scintillation Crystals, Energoatomizdat, Moscow 1991 (in Russian).

[31] I.M. Solskii, Tekhnologiya i konstruirovanie $v$ elektronnoi apparature 6, 47 (2005) (in Russian).

[32] F.A. Danevich, I.K. Bailiff, V.V. Kobychev, H. Kraus, M. Laubenstein, P. Loaiza, V.B. Mikhailik, S.S. Nagorny, A.S. Nikolaiko, S. Nisi, I.M. Solsky, G. Warot, Nucl. Instrum. Meth. Phys. Res. A 631, 44 (2011).

[33] Yu.A. Borodenko, B.V. Grinyov, V.P. Martynov, L.A. Piven, I.M. Solskii, Yu.V. Zorenko, Z.T. Moroz, M.V. Pashkovskii, Surface. X-ray, Synchrotron, and Neutron Investigations 6, 6 (2002) (in Russian).

[34] J.D. Vergados, H. Ejiri, F. Šimkovic, Rep. Prog. Phys. 75, 106301 (2012).
[35] A.N. Annenkov, O.A. Buzanov, F.A. Danevich, A.Sh. Georgadze, S.K. Kim, H.J. Kim, Y.D. Kim, V.V. Kobychev, V.N. Kornoukhov, M. Korzhik, J.I. Lee, O. Missevitch, V.M. Mokina, S.S. Nagorny, A.S. Nikolaiko, D.V. Poda, R.B. Podviyanuk, D.J. Sedlak, O.G. Shkulkova, J.H. So, I.M. Solsky, V.I. Tretyak, S.S. Yurchenko, Nucl. Instrum. Meth. Phys. Res. A 584, 334 (2008).

[36] S. Pirro, J.W. Beeman, S. Capelli, M. Pavan, E. Previtali, P. Gorla, Phys. At. Nucl. 69, 2109 (2006).

[37] S.J. Lee, J.H. Choi, F.A. Danevich, Y.S. Jang, W.G. Kang, N. Khanbekov, H.J. Kim, I.H. Kim, S.C. Kim, S.K. Kim, Y.D. Kim, Y.H. Kim, V.V. Kobychev, V.N. Kornoukhov, J.I. Lee, J.S. Lee, K.B. Lee, M.K. Lee, Y.H. Lee, S.S. Myung, J.H. So, V.I. Tretyak, Y. Yuryev, Astropart. Phys. 34, 732 (2011).

[38] H.J. Kim, A.N. Annenkov, R.S. Boiko, O.A. Buzanov, D.M. Chernyak, J.H. Cho, F.A. Danevich, A.E. Dossovitsky, Gul Rooh, U.K. Kang, M.J. Kim, S.C. Kim, S.K. Kim, Y.D. Kim, V.V. Kobychev, V.N. Kornoukhov, M.B. Kosmyna, S.J. Lee, J.I. Lee, J.H. Lee, S.S. Myung, B.P. Nazarenko, A.S. Nikolaiko, R.B. Podviyanuk, V.M. Puzikov, A.N. Shekhovtsov, J.H. So, I. Solskii, V.I. Tretyak, A.V. Veresnikova, IEEE Trans. Nucl. Sci. 57, 1475 (2010).

[39] www.goochandhousego.com .

[40] www.yamajuceramics.co.jp .

[41] www.jsgd.en.ec21.com .

[42] www.ikz-berlin.de .

[43] www.crytur.cz .

[44] www.itme.edu.pl. 